\title{
CHARGE TRANSFER STATES OF TRANSITION METAL AND RARE EARTH IONS: THEIR ROLE IN RECOMBINATION PROCESSES
}

\author{
M. GoDLEWSKI \\ Institute of Physics, Polish Academy of Sciences \\ Al. Lotników 32/46, 02-668 Warszawa, Poland
}

\begin{abstract}
Center ionization accompanied by carrier localization at the center by virtue of a short-range or a long-range Coulomb attractive potential is discussed. The role of charge transfer states in recombination processes is described. The source of carrier attractive potential is explained and a simple theoretical model is presented allowing to predict the nature (either electron or hole attractive) of this potential. The probability of different possible recombination paths is also analyzed.
\end{abstract}

PACS numbers: 71.35.+z, 78.55.Cr, 78.55.Et

\section{Introduction}

The main hindrance, as far as the application of rare-earth (RE) activated materials is concerned, is the low efficiency of the parity forbidden $4 f-4 f$ emission. Screening by electrons from external $5 s$ and $5 p$ orbits preserves a nearly atomic character of the $4 f$ shell resulting on one hand in sharp, temperature and environment independent emissions, but on the other hand it leads to low excitation and deexcitation efficiencies. Even though the $4 f-4 f$ transition can also be induced by coherent or incoherent energy transfer via electron-hole pairs [1], the efficiency of such excitation is rather low.

Recently new, more efficient excitation channels were predicted for those of the RE ions which can either change their charge state or can localize, by virtue of a short-range or a long-range Coulomb potential, free carriers [2-4]. In the latter case a charge transfer state of the RE or transition metal (TM) ion is formed, consisting of the ion with an externally bound carrier. Rich experimental evidence was collected proving that such states may be quite common. They were observed not only for several TM and RE ions [4], but also for typical isoelectronic defects in semiconductors [5]. 
The possible recombination channels of TM and RE ions, including carrier binding via the charge transfer states, will be discussed in this work. It will be shown that quite complicated recombination paths may result. However, they can have very different transition probabilities. Some simple predictions concerning the nature of the short-range carrier binding potential, as well as the efficiencies of different recombination paths will be given. Also, some unsolved theoretical aspects will be pointed out.

\section{Carrier binding by RE and TM ions}

Carrier binding can be easily understood in the case of impurities which can change their oxidation state. Once the impurity is ionized, it becomes charged in respect to the lattice sites and may attract a free carrier by a long-range Coulomb potential. For neutral (isoelectronic) impurities the carrier binding must be of a different origin. The appropriate mechanism was proposed by Thomas [6] and may be of identical nature to that responsible for the primary carrier binding in the case of excitons bound on isoelectronic centers [7]. The carrier is trapped by a short-range potential caused either by a difference between the electronegativities of the impurity and the ion for which it substitutes and/or lattice distortion induced by the substitution [8]. Core states polarization induced by the trapped carrier may also contribute to the binding potential introducing a long-range component to the attractive potential. The core polarization may also enhance the subsequent energy transfer to the impurity core states, as will be discussed further on.

The short-range binding potential of the ion may be either electron or hole attractive. A simple method for predicting the kind of the carrier binding potential was proposed by Robbins and Dean [9] for carrier binding by TM ions and will be adapted here to describe the properties of the RE impurities. If an ion in its neutral charge state introduces an energy level into the band gap of the host material, i.e., it can change its charge state from the neutral to the positively charged in respect to the host atom, it is expected to have a short-range hole attractive potential. The creation of the short-range hole attractive potential may also occur for those TM or RE ions which have only an unfilled atomic level within the band gap of the material. This is due to the higher order interactions admixing the ground state of the ion to the excited band gap state, i.e., the ground state of the neutral ion "partly enters" into the band gap. On the other hand, if only the negatively charged ion introduces an energy level into the band gap, the center may localize an electron by virtue of a short-range potential.

Using the above model, we can predict that a hole binding may occur in the case of $\mathrm{Ce}^{3+}, \mathrm{Pr}^{3+}, \mathrm{Tb}^{3+}$, or $\mathrm{Eu}^{2+}$ and an electron binding e.g. for $\mathrm{Yb}^{3+}$ and $\mathrm{Eu}^{3+}$. This is rather a tentative model but it has already found some indirect experimental confirmation. We indicate also that some of the TM or RE ions, which may occur in different charge states may bind, by virtue of a short-range or a long-range Coulomb potential, either electron or hole depending on initial oxidation state. This is very different from the case of common isoelectronic centers in semiconductors. 


\section{Notation}

To simplify the discussion the following notation will be used. $[I],\left[I^{+}\right],\left[I^{-}\right]$ stand for the neutral, positive and negative charge states of the ion, in respect to the lattice site. $\left[I^{*}\right]$ denotes an excited core state of the neutral ion and a similar notation will be used for the other charge states. $[I, \mathrm{e}]$ and $[I, \mathrm{~h}]$ describe a neutral ion with an electron or hole localized by a short-range potential, respectively. $[I]_{\mathrm{h}}^{\mathrm{e}}$ is a neutral impurity bound exciton state, whereas $\left[I^{-}\right], \mathrm{h}$ or $\left[I^{+}\right], \mathrm{e}$ denote the ionized ion with a carrier localized by a Coulomb attractive potential of the center. Other combinations of these different states are possible but their notation may be understood basing on the above given examples.

It should be mentioned that such impurity states are known in the literature under different names. For example, the ion with a free carrier localized either by a short-range or the Coulomb potential is described in some papers as a localized impurity bound exciton state $[3,4,9]$, a charge transfer state [10] or a transient donor (acceptor) state [11]. This is rather confusing. In this paper such a state will be called a charge transfer state and the bound exciton notation will be used to describe the resulting recombination transition of the localized carrier with one tightly bound at impurity core.

\section{Photoluminescence excitation mechanisms}

We will discuss now the possible recombination channels occurring after carrier binding by a TM or RE ion. Let us assume that the ion possesses a hole attractive potential, i.e., it may localize a free hole induced in the valence band (VB) by the initial band to band excitation

$$
[I]+\mathrm{e}, \mathrm{h} \rightarrow[I, \mathrm{~h}]+\mathrm{e} \text {. }
$$

Such a process results in the formation of a charge transfer state of the impurity. The $[I, \mathrm{~h}]$ excited state of the ion is not stable and after its formation three different situations can occur. The first possibility is the localization of a second carrier, in this case an electron, by the Coulomb attractive potential formed at the ion after binding of the first carrier

$$
[I, \mathrm{~h}]+\mathrm{e} \rightarrow[I]_{\mathrm{h}}^{\mathrm{e}} \text {. }
$$

The second alternative is that the externally bound hole will recombine with an electron on the core state of the ion with an energy transfer to the ion core states. Such a process is called the defect Auger recombination [9] and is sometimes described in the literature as a nonradiative recombination of a localized bound exciton (LBE) state of the ion

$$
[I, \mathrm{~h}]+\mathrm{e} \rightarrow\left[I^{+*}\right]+\mathrm{e} \text {. }
$$

In some cases a localized bound exciton may decay radiatively resulting in a new ion related emission

$$
[I, \mathrm{~h}]+\mathrm{e} \rightarrow\left[I^{+}\right]+\mathrm{e}+h \nu_{\mathrm{LBE}} \text {. }
$$

Each recombination step listed above may be followed by further transitions. In the first case the direct bound exciton (BE) emission can occur

$$
[I]_{\mathrm{h}}^{\mathrm{e}} \rightarrow[I]+h \nu_{\mathrm{BE}} \text {. }
$$


The defect Auger recombination, i.e., nonradiative $\mathrm{BE}$ recombination due to an energy transfer to core states of an ion, may also be expected

$$
[I]_{\mathrm{h}}^{\mathrm{e}} \rightarrow\left[I^{*}\right]+\Delta E \text {. }
$$

The creation and subsequent recombination of a bound exciton state leave the impurity ion in its final, ground state. On the contrary, the process described by formulae (3) and (4) ends with the positive charge state of the ion, which is still an excited state. The following recombination processes of this state with a free electron may also proceed on very different ways. Radiative or nonradiative electron capture may occur

$$
\left[I^{+}\right]+\mathrm{e} \rightarrow[I]+\Delta E \text {. }
$$

Otherwise, the carrier may be bound via a charge transfer state

$$
\left[I^{+}\right]+\mathrm{e} \rightarrow\left[I^{+}\right], \mathrm{e}+\Delta E
$$

or via a core excited state of the ion

$$
\left[I^{+}\right]+\mathrm{e} \rightarrow\left[I^{*}\right]+\Delta E \rightarrow[I]+h \nu_{\text {intra-shell }} \text {. }
$$

Finally, the charge transfer state may decay via a localized BE emission

$$
\left[I^{+}\right], \mathrm{e} \rightarrow[I]+h \nu_{\mathrm{LBE}},
$$

or may convert into an excited core state of the ion by the defect Auger recombination

$$
\left[I^{+}\right], \mathrm{e} \rightarrow\left[I^{*}\right]+\Delta E \rightarrow[I]+h \nu_{\text {intra-shell }} .
$$

The determination of the actually occurring recombination paths is difficult, since often the same emission is obtained.

\section{Predictions on recombination efficiency}

The recombination patterns described above seem at first view to be very complicated. Once we include the possibility of carrier localization, many new recombination channels are opened and they may compete with each other. However, there are some simple rules which can help us to predict the most probable scenarios. Among the three possible paths (mentioned above) following hole localization by a short-range potential, i.e., the formation of a charge transfer state, the defect Auger recombination is the most likely. The extremely high efficiency of this process was verified experimentally. Podlowski et al. [12] observed a totally nonradiative decay of a localized $\mathrm{BE}$ for the $\mathrm{Ni}^{2+}$ ion in $\mathrm{ZnS}$ and $\mathrm{CdS}$ via the Auger type energy transfer mechanism $\left(\left[\mathrm{Ni}^{1+}\right], \mathrm{h} \rightarrow \mathrm{Ni}^{2+, *}\right)$. However, this is true for those ions which have at least one of the excited core states quasi-resonant with the charge transfer state. If the energy mismatch between LBE and core transitions is large, the efficiency of the Auger process is reduced and radiative recombination of the localized BE becomes dominant. This was, for example, observed for the copper impurity in the $\mathrm{ZnO}$ lattice [11]. Energy transfer from the BE state to core states of the $\mathrm{Cu}$ ion would require dissipation (by phonon emission) of a large amount of the energy making this process inefficient. A unique situation was found recently for the $\mathrm{Yb}$ ion in $\mathrm{ZnS}$. Because of the large energy mismatch between the 
LBE $\left(\left[\mathrm{Yb}^{2+}, \mathrm{h}\right]\right)$ and $\mathrm{Yb}^{3+}$ intra-shell transition the defect Auger recombination was of reduced efficiency and both LBE and $4 f-4 f$ transitions could be observed simultaneously [13].

In some rare cases the charge transfer state was found to be the lowest excited state of the ion. Then, only the LBE emission was observed. This may occur for such TM or RE ions which have large energy differences between their ground and excited states. Thus, it is expected for ions with either half filled or closed $3 d$ or $4 f$ shells. The LBE emission was observed e.g. for $\mathrm{Yb}^{2+}$ in $\mathrm{SrF}_{2}$ and $\mathrm{Eu}^{2+}$ in $\mathrm{BaF}_{2}$ $[14,15]$.

In agreement with the above reasoning the ion intra-shell emission will occur when the excited state of the core is quasi-resonant with the charge transfer state. The localized BE emission will occur either if there is a large energy mismatch between the charge transfer state and the lower lying core states of the ion or if the charge transfer state is the lowest excited energy state of the ion. This point was carefully tested for the europium ion in the wide band gap II-VI compounds. For $\mathrm{Eu}^{2+}$ ion in $\mathrm{ZnS}$ a charge transfer state $\left(\left[\mathrm{Eu}^{3+}\right], \mathrm{e}\right.$, which is the equivalent of the $\left[I^{+}\right]$,e state) is the lowest excited state of the ion and only the localized $\mathrm{BE}$ emission was observed [16]. For $\mathrm{Eu}^{2+}$ in $\mathrm{CaS}$ the $4 f^{6} 5 d^{1} \rightarrow 4 f^{7}$ emission is quasi-resonant with the LBE transition and was observed in photoluminescence (PL) [17].

In some cases binding of a carrier by a TM or RE ion may be followed by localization of a second carrier (Eq. (2)), which is analogous to the formation of the well-known isoelectronic center bound exciton. This scenario is, however, less probable and will occur only if the lifetime of the charge transfer state is sufficiently long. Practically, it may occur only if the processes (3) and (4) cannot be realized, i.e., when the ion cannot change its charge state. The exciton binding and its direct radiative recombination was observed recently for $\mathrm{Yb}^{3+}$ and $\mathrm{Er}^{3+}$ ions in the InP lattice [18-20]. In both these cases the 2+ charge state of the $\mathrm{RE}$ ion does not give rise to an energy level in the forbidden band gap of InP [21]. Thus, the electron localization on the charge transfer state could not be followed by the formation of the $2+$ excited charge state of $\mathrm{Yb}$ and Er. Consequently, the favorable condition for exciton binding was realized.

As already mentioned, once a bound exciton or a localized bound exciton state is formed, the defect Auger recombination is the most likely recombination path. Only in some "special" cases direct radiative recombination of such states was observed in the photoluminescence. This means that carrier binding by TM or RE ions results in most of the cases only in their intra-shell transitions, i.e., the same emissions as observed under direct $4 f-4 f$ excitation. However, the excitation efficiency in the former case may be much higher since, e.g., core polarization induced by the externally bound carrier may lead to some admixture of higher lying core states to the ground one and, thus, to a relaxation of the selection rules.

Still, because of the similarity of the observed emissions the formation of the BE state may, in most of the cases, be concluded only indirectly. This can be done either with the use of the photoluminescence excitation (PLE) spectroscopy $[12,13,22,23]$ or by observation of the characteristic temperature dependence of 
the PL and PLE efficiencies [13]. This also explains why the processes discussed in the present paper were not taken into account until very recently.

\section{Hypotheses}

As already mentioned, the existence of charge transfer states of TM and RE ions is not easy to confirm experimentally. There are several indirect experimental evidences that excitation processes of the type discussed in this paper are of practical importance. However, their knowledge is still rather inadequate.

Some fundamental questions are still not answered. For example, it would be interesting to know whether the charge transfer states could influence the cross-section of photoionization transitions of the RE ions. The charge transfer state is quasi-resonant with the edge of the photoionization transition. Thus, it may happen that the first step in the photoionization transition is the excitation to the charge transfer state, which is then followed by carrier emission to the continuum of the band states. There are some indications that such a scheme may take place. For example, it was observed that both ionization transitions of the $\mathrm{Yb}$ ion in $\mathrm{ZnS}$ (valence band $\rightarrow \mathrm{Yb}^{2+}$ and $\mathrm{Yb}^{2+} \rightarrow$ conduction band) are best described by formulae appropriate for parity allowed processes [13], which is certainly not expected for the direct RE ion $\rightarrow$ conduction band process. However, it may be explained once we include the co-existence of the charge transfer state.

The electronic structure of charge transfer states was revealed only in a very few cases [10, 24-29]. However, the conclusions derived from different magnetic resonance or magneto-optical experiments are often not consistent. We would like to indicate, for example, that the formation of an ionized acceptor bound exciton was concluded by Hoffmann et al. [26], which is not expected for ionized shallow acceptors in any of the common semiconductors. It is a matter of further studies to confirm the correctness of such identifications.

This work was partly supported by the research grant no. 204769101 of the Committee for Scientific Research.

\section{References}

[1] S. Schmitt-Rink, C.M. Varma, A.F.J. Levi, Phys. Rev. Lett. 66, 2782 (1991).

[2] K. Świątek, A. Suchocki, M. Godlewski, Appl. Phys. Lett. 56, 195 (1990).

[3] M. Godlewski, K. Świątek, A. Suchocki, J.M. Langer, J. Lumin. 48/49, 23 (1991).

[4] M. Godlewski, K. Świątek, J. Cryst. Growth 117, 634 (1992).

[5] J. Wagner, R. Sauer, Phys. Rev. B 26, 3502 (1982).

[6] D.G. Thomas, J. Phys. Soc. Jpn. (Suppl.) 21, 265 (1966).

[7] J.J. Hopfield, D.G. Thomas, R.T. Lynch, Phys. Rev. Lett. 17, 312 (1966).

[8] W. Czaja, in: Advances in Solid State Physics XI, Ed. O. Madelung, Pergamon Press, Oxford 1971, p. 65.

[9] D.J. Robbins, P.J. Dean, Adv. Phys. 27, 499 (1978).

[10] D.J. Robbins, J. Lumin. 24/25, 137 (1981). 
[11] R. Dingle, Phys. Rev. Lett. 23, 579 (1969).

[12] L. Podlowski, R. Heitz, A. Hoftmann, I. Broser, J. Lumin. 53, 401 (1992).

[13] H. Przybylińska, K. Świątek, A. Stąpor, A. Suchocki, M. Godlewski, Phys. Rev. B 40, 1748 (1989).

[14] D.S. McClure, C. Pedrini, Phys. Rev. B 32, 8465 (1985).

[15] B. Moine, B. Courtois, C. Pedrini, J. Phys. (France) 50, 2105 (1989).

[16] K. Świątek, M. Godlewski, D. Hommel, Phys. Rev. B 42, 3628 (1990).

[17] K. Świątek, A. Suchocki, M. Godlewski, in: The Second Int. School on Excited States of Transition Elements, Karpacz (Poland) 1991, Eds. W. Stręk, W. RybaRomanowski, J. Legendziewicz, B. Jeżowska-Trzebiatowska, World Scientific, Singapore 1992, p. 421.

[18] B.J. Heijmink Liesert, M. Godlewski, A. Stąpor, T. Gregorkiewicz, C.A.J. Ammerlaan, J. Weber, M. Moser, F. Scholz, Appl. Phys. Lett. 58, 2237 (1991).

[19] K. Thonke, K. Pressel, G. Bohnert, A. Stąpor, J. Weber, M. Moser, A. Malassioti, A. Hangleiter, F. Scholz, Semicond. Sci. Technol. 5, 1124 (1990).

[20] B. Lambert, A. Le Corre, Y. Toudic, C. Lhomer, G. Grandpierre, M. Gauneau, J. Phys., Condens. Matter 2, 479 (1990).

[21] C. Delerue, M. Lannoo, Phys. Rev. Lett. 67, 3006 (1991).

[22] A. Hoffmann, B. Lummer, Ch. Fricke, R. Heitz, I. Broser, J. Cryst. Growth 117, 640 (1992).

[23] R. Heitz, A. Hoffmann, P. Thurian, I. Broser, J. Phys. Condens. Matter 4, 157 (1991).

[24] J. Schneider, U. Kaufmann, W. Wilkening, M. Baeumler, F. Köhl, Phys. Rev. Lett. 59, 240 (1987).

[25] M. Godlewski, W.E. Lamb, B.C. Cavenett, J. Phys. C 15, 3925 (1982).

[26] A. Hoffmann, A. Franz, A. Ismail, F. Asch, I. Broser, Mater. Sci. Forum 38-41, 525 (1989).

[27] H. Kawai, T. Hoshina, Solid State Commun. 22, 391 (1977).

[28] I.J. Broser, R.K.F. Germar, H.-J.E. Schulz, K.P. Wisznewski, Solid State Electron. 21, 1597 (1978).

[29] P.J. Dean, D.J. Robbins, S.G. Bishop, J.A. Savage, P. Porteous, J. Phys. C 14, 2847 (1981). 